\title{
Correction to: Effectiveness of immediate vitrectomy and intravitreal antibiotics for post-injection endophthalmitis
}

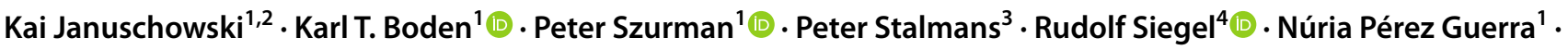 \\ Sören L. Becker ${ }^{5}$ (D) Annekatrin Rickmann ${ }^{1}\left[\right.$ ] . Lukas Bisorca-Gassendorf ${ }^{1}[\mathbb{C}$
}

(c) Springer-Verlag GmbH Germany, part of Springer Nature 2021

\section{Correction to: Graefe's Archive for Clinical and Experimental Ophthalmology https://doi.org/10.1007/s00417-021-05071-w}

The original publication of this paper contains inaccuracies in the key messages.

The authors prefer some short and on point statements, such as:

\section{Key messages}

- Endophthalmitis is a sight-threatening postoperative complication.

- Immediate PPV with subsequent intravitreal injection of antibiotics can restore BCVA.

- PPV should be carried out as early as possible, where available.

The original article has been corrected.

Publisher's note Springer Nature remains neutral with regard to jurisdictional claims in published maps and institutional affiliations.

The online version of the original article can be found at https:// doi.org/10.1007/s00417-021-05071-w

Lukas Bisorca-Gassendorf

lukas.bisorca-gassendorf@kksaar.de

1 Eye Clinic Sulzbach, Knappschaft Hospital Saar, An der Klinik 10, 66280 Sulzbach, Germany

2 Centre for Ophthalmology, University Eye Hospital Tuebingen, Schleichstraße 12, 72076 Tuebingen, Germany

3 Department of Ophthalmology, University Hospitals Leuven, Herestraat 49, 3000 Leuven, Belgium

4 Industrial and Organizational Psychology, Saarland University, Campus A1.3, 66123 Saarbruecken, Germany

5 Institute of Medical Microbiology and Hygiene, Saarland University, Kirrberger Straße, Building 43, 66421 Homburg, Germany 